\title{
Corrigendum
}

\section{Corrigendum to "Multistage System-Based Machine Learning Techniques for Intrusion Detection in WiFi Network"}

\author{
Vu Viet Thang $\mathbb{D}^{1,2}$ and F. F. Pashchenko ${ }^{3,4}$ \\ ${ }^{1}$ Moscow Institute of Physics and Technology (State University), Moscow, Russia \\ ${ }^{2}$ Faculty of Information Technology, Hanoi University of Industry, Hanoi, Vietnam \\ ${ }^{3}$ The Department of Information and Communication Technologies, MIPT (State University), Moscow, Russia \\ ${ }^{4}$ Trapeznikov Institute of Control Sciences, Russian Academy of Sciences, Moscow, Russia \\ Correspondence should be addressed to Vu Viet Thang; vv.tkhang@phystech.edu \\ Received 9 August 2020; Accepted 9 August 2020; Published 8 September 2020 \\ Copyright ( $92020 \mathrm{Vu}$ Viet Thang and F. F. Pashchenko. This is an open access article distributed under the Creative Commons \\ Attribution License, which permits unrestricted use, distribution, and reproduction in any medium, provided the original work is \\ properly cited.
}

In the article titled "Multistage System-Based Machine Learning Techniques for Intrusion Detection in WiFi Network" [1], there was a missing affiliation for the second author. The corrected authors' list and affiliations are shown above.

\section{References}

[1] V. V. Thang and F. F. Pashchenko, "Multistage system-based machine learning Techniques for intrusion detection in WiFi Network," Journal of Computer Networks and Communications, vol. 2019, Article ID 4708201, 13 pages, 2019. 